\title{
Classical Hodgkin Lymphoma Presenting as the Primary Rectal Lesion in a HIV patient on HAART Management
}

\author{
Hariharanadha Sarma $\mathrm{N}^{1}$, Chaitanya B ${ }^{1}$, Saiswaroop $\mathrm{G}^{2}$, Manu Goyal ${ }^{3}$ \\ ${ }^{1}$ Departments of Anatomic Pathology and ${ }^{2}$ General \& Minimal Invasive Surgery, RDT hospital, Bathalapalli -515661, Andhra \\ Pradesh; ${ }^{3}$ Department of Hematopathology, AMPATH, Hyderabad-500012, Telengana.
}

\section{Corresponding Author: \\ Dr. Hariharanadha Sarma.N \\ Email: nandyalahari@yahoo.com}

This is an Open Access article distributed under the terms of the Creative Commons Attribution License (creativecommons.org/ licenses/by/3.0).

Published

August 30, 2019

\begin{abstract}
Background: Hodgkin lymphoma involving rectum as primary lesion is very rare. Incidence rates of Hodgkin lymphoma have increased by 7-8 times in HIV patients when compared with the general population. The incidence has increased in the era of highly active anti-retroviral therapy (HAART). Only five cases of primary Hodgkin lymphoma of the rectum are reported in the literature. Case Report: We are presenting a case of primary Hodgkin lymphoma of the rectum in a HIV infected patient on HAART. Conclusion: Hodgkin lymphoma involving the rectum as a primary lesion is very rare and in HIV infected patients is much rarer. Hodgkin lymphoma should be kept in mind in an anorectal growth in HIV patients.
\end{abstract}

Keywords: HIV Infections, Highly Active Antiretroviral Therapy, Hodgkin Disease, Lymphoma, Rectum.

\section{Introduction}

Classic Hodgkin lymphoma (CHL) is a clonal malignant lymphoproliferation originating from germinal centre B cells [1]. The risk for developing CHL has increased 6-20 folds for human immunodeficiency virus (HIV) infected persons. The incidence of CHL seems to increase under highly active anti retroviral therapy (HAART). Hodgkin lymphoma is the most common type of non-AIDS-defining tumor that occurs in the HIV population. CD4 cell counts are relatively high at presentation compared with those in non-Hodgkin lymphomas. Primary gastrointestinal lymphomas are very rare [2]. Primary CHL in the rectum is much rarer and is limited to a few case reports so far. Primary CHL in the rectum in HIV infected persons has been a rarity with only five cases described in the literature so far. The recently described lesion "EBV positive mucocutaneous ulcer (EBVPMU) has to be excluded in gastrointestinal tract (GIT) lesion because of similar histological features. We report a case of CHL primarily affecting the rectum in a HIV patient on HAART.

\section{Case Report}

A 39 year old female presented to the outpatient department of surgery with the complaints of blood in the stools for the last one month. She is a known HIV positive person and has been on HAART for the last two years at our antiretroviral therapy (ART) centre. Physical examination revealed an ulcerated growth in the anorectal junction which was seen extending on to the rectal wall. The growth was indurated, obstructive and measured $2 \times 3 \mathrm{cms}$ in size. Thorough clinical examination did not reveal any other significant findings. There were no other obvious swellings anywhere in her body. The growth was suspected to be carcinoma and an incisional biopsy was taken. The tissue was sent to pathology for confirmation.

The specimen was a greyish brown soft tissue measuring $1 \times 1 \times 2 \mathrm{~cm}^{3}$. Histopathology 
revealed scattered sheets or nodular clusters of lymphocytes, plasma cells, eosinophils and occasional large binucleated cells with prominent nucleolus (Reed-Sternberg cell). There were some large cells with hyperchromatic condensed nuclei (mummified R-S cells) [Fig.1]. Classical Hodgkin lymphoma was diagnosed and the tissue was sent to AMPATH Laboratories, Hyderabad for immunohistochemistry (IHC) staining and confirmation of the diagnosis. The IHC stains CD30, CD15, Pax5 and EBER were positive to confirm the diagnosis of CHL [Fig.2].

The patient was referred to a specialized oncocentre for the management. The patient has been followed up for one year and she is doing well without any complications.

\section{Discussion}

Primary colorectal lymphomas account for less than $1 \%$ of colorectal malignancies [2]. The criteria for primary gastrointestinal (GI) lymphomas include the absence of superficial lymphadenopathy at the time of diagnosis, absence of peritoneal or mediastinal lymphadenopathy, normal blood counts, absence of hepatic or splenic infiltrates and predominance of GI lesion at the time of diagnosis [3]. Hodgkin lymphoma (HL) is a lymph nodal disease with rare encounters of extra-nodal forms. GIT is the most common extranodal site followed by lung, thyroid, skin, genitourinary system and central nervous system [4]. Palpable abdominal mass and weight loss can be the presenting symptoms in GIT lymphomas. Diagnosis of colorectal lymphomas requires a high index of suspicion due to its rarity and non-specific clinical and diagnostic signs.

HIV infected persons have a 6-20 fold increased risk of developing CHL. Incidence of CHL seems to increase under HAART management in HIV infected persons. In acquired immuno deficiency syndrome (AIDS) setting CHL shows a predominance of unfavourable histologies and

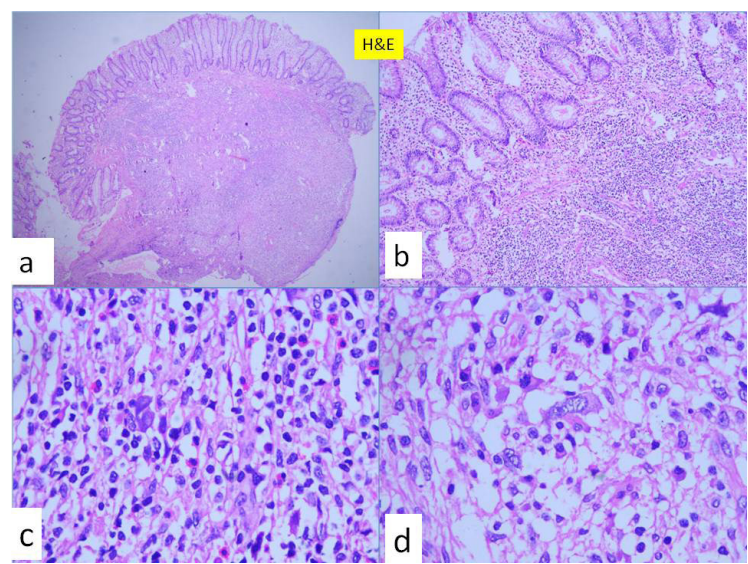

Fig.1: $H \&$ E section (a): Scanner view of the tumor in the submucosa seen as vague nodular growth $\times 40$. (b): Low power view of the tumour in the submucosa with normal mucosa above $\times 100$. (c \& d): High power view of the tumour showing lymphocytes, eosinophils, classical and mummified Reed-Sternberg $(R-S)$ cells $\times 400$.

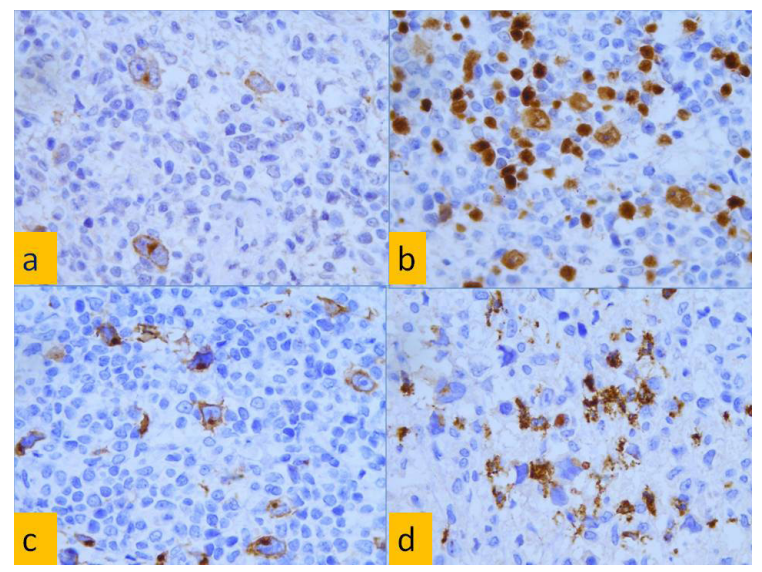

Fig.2: $I H C$ (a): $R-S$ cells showing CD15 positivity. (b): $R-S$ cells showing pax 5 positivity of $B$-lymphocytes and $R-S$ cells. (c): $R-S$ cells showing CD30 positivity. (d): EBER scattered positivity in $R-S$ cells.

is almost universally associated with Ebstein-Barr virus (EBV). Another suggestion of infectious etiology comes from the higher incidence of HL in HIV positive persons. In this population, HL has a stronger association with EBV than it does among the HIV negative persons. The fall in the incidence of HL as CD4 counts fall also suggests a role of a dysregulated immunological response in the development of the disease [5]. 
Simpson et al. [6] found five cases of primary rectal HL in HIV positive persons. Mean patient age was 39 years, all were male and four were homosexual. All of them had CHL features, four of them presenting in stage IV. Valbuenna et al. [7] identified 12 cases of primary HL of the rectum in immunocompetent persons from the literature. Only one out of these 12 persons were tested positive for EBV and only two had confirmation of the diagnosis by immunohistochemistry. In the present case the person is a 39 year old female who is a known HIV positive and has been on HAART for the last two years. This person did not have any other manifestations in the body to suggest Hodgkin lymphoma.

Hodgkin lymphoma of the rectum has to be differentiated from a recently described lesion "EBV positive mucocutaneous ulcer (EBVMCU). This lesion has many features similar to HL. This EBV positive mucocutaneous ulcer shows shallow, well demarcated ulcers involving the mucosa of the oropharynx, GIT and skin [8]. Immunosenescence due to aging and immunosuppressive usage can lead to this condition. This lesion has indolent course and requires conservative management unlike Hodgkin lymphoma.

\section{Conclusion}

Hodgkin lymphoma of the rectum is a rare lesion either in HIV affected persons or in immunocompetent persons. We conclude that Hodgkin lymphoma should be kept in mind whenever an obstructive lesion is seen in the rectum especially in HIV affected persons.
Contributors: HSN: Collection of case details, search data base for more information about the case and references and preparation of manuscript; CB: editing the manuscript, collection of references about other reported data; SSG: providing all the clinical details of the case and management details; MG: performing the IHC on the case and giving the final comment on the case. HSN will act as guarantor of the study. All authors approved the final version of this article. Funding: None; Competing interests: None stated.

\section{References}

1. Fend F. Classical Hodgkins lymphoma. In: Jaffe ES, Harris NL, Vardiman JW, Campo E, Arber DA. Hematopathology. St.Louis: Saunders; 2011: pp.454472.

2. Fan CW, Changchien CR, Wang JY, Chen JS, Hsu KC, Tang R, et al. Primary colorectal lymphoma. Dis Colon Rectum. 2000;43:1277-1282.

3. Dawson IM, Cornes JS, Morson BC. Primary malignant lymphoid tumors of the intestinal tract. Report of 37 cases with a study of factors influencing the prognosis. Br J Surg. 1961;49:80-89.

4. Gandhi JS, Mehta A, Sharma A, Kamboj M. Primary Hodgkin lymphoma of the ileum. J Cancer Res Ther. 2010;6:342-343.

5. Ambinder RF. Epstein-Barr virus and Hodgkin lymphoma. Hematology Am Soc Hematol Educ Program. 2007:204-209.

6. Simpson L, Taylor S, Piotrowski A. Uncommon presentations of Hodgkin's disease. Case 3 Hodgkin's disease, Rectal presentation. J Clin Oncol. 2004;22:196198.

7. Valbuena JR,Gualco G, Espejo-Plascencia I, Medeiros LJ. Classical Hodgkin lymphoma arising in rectum. Ann Diag Pathol. 2005;9:38-42.

8. Dojcinov SD, Venkataraman G, Raffeld M, Pittaluga $\mathrm{S}$, Jaffe ES. EBV positive mucocutaneous ulcer- a study of 26 cases associated with various sources of immunosuppression. Am J Surg Pathol. 2010;34:405417. 